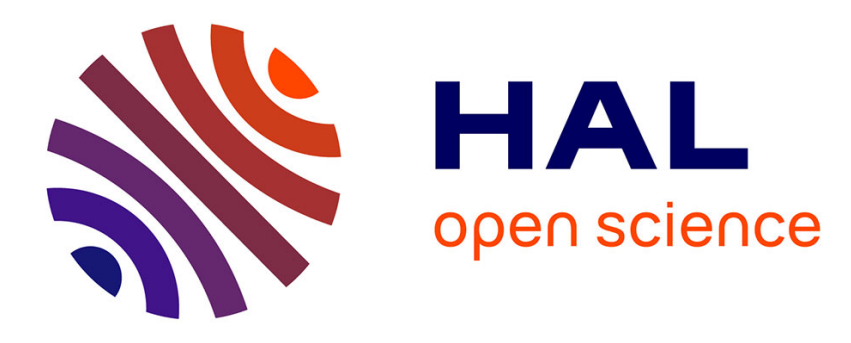

\title{
Dynamics and bifurcations of the adaptive exponential integrate-and-fire model
}

\author{
Jonathan Touboul, Romain Brette
}

\section{To cite this version:}

Jonathan Touboul, Romain Brette. Dynamics and bifurcations of the adaptive exponential integrateand-fire model. [Research Report] RR-6563, INRIA. 2008. inria-00288734v4

\section{HAL Id: inria-00288734 https://hal.inria.fr/inria-00288734v4}

Submitted on 2 Jul 2008

HAL is a multi-disciplinary open access archive for the deposit and dissemination of scientific research documents, whether they are published or not. The documents may come from teaching and research institutions in France or abroad, or from public or private research centers.
L'archive ouverte pluridisciplinaire HAL, est destinée au dépôt et à la diffusion de documents scientifiques de niveau recherche, publiés ou non, émanant des établissements d'enseignement et de recherche français ou étrangers, des laboratoires publics ou privés. 
INSTITUT NATIONAL DE RECHERCHE EN INFORMATIQUE ET EN AUTOMATIQUE

\title{
Dynamics and bifurcations of the adaptive exponential integrate-and-fire model
}

\author{
Jonathan Touboul — Romain Brette
}

\section{$N^{\circ} 6563$}

July 2, 2008

Thème $\mathrm{BIO}$

\section{apport}

derecherche 



\title{
Dynamics and bifurcations of the adaptive exponential integrate-and-fire model
}

\author{
Jonathan Touboul, Romain Brette * \\ Thème BIO — Systèmes biologiques \\ Projet Odyssée ${ }^{\dagger}$ \\ Rapport de recherche $n^{\circ} 6563$ - July 2, 2008 - 28 pages
}

\begin{abstract}
Recently, several two-dimensional spiking neuron models have been introduced, with the aim of reproducing the diversity of electrophysiological features displayed by real neurons while keeping a simple model, for simulation and analysis purposes. Among these models, the adaptive integrate-and-fire model is physiologically relevant in that its parameters can be easily related to physiological quantities. The interaction of the differential equations with the reset results in a rich and complex dynamical structure. We relate the subthreshold features of the model to the dynamical properties of the differential system and the spike patterns to the properties of a Poincaré map defined by the sequence of spikes. We find a complex bifurcation structure which has a direct interpretation in terms of spike trains. For some parameter values, spike patterns are chaotic.
\end{abstract}

\section{Key-words:}

* brette@di.ens.fr

$\dagger$ Odyssée is a joint project between ENPC - ENS Ulm - INRIA 


\section{Dynamics and bifurcations of the adaptive exponential integrate-and-fire model}

Résumé : Recently, several two-dimensional spiking neuron models have been introduced, with the aim of reproducing the diversity of electrophysiological features displayed by real neurons while keeping a simple model, for simulation and analysis purposes. Among these models, the adaptive integrate-and-fire model is physiologically relevant in that its parameters can be easily related to physiological quantities. The interaction of the differential equations with the reset results in a rich and complex dynamical structure. We relate the subthreshold features of the model to the dynamical properties of the differential system and the spike patterns to the properties of a Poincaré map defined by the sequence of spikes. We find a complex bifurcation structure which has a direct interpretation in terms of spike trains. For some parameter values, spike patterns are chaotic.

Mots-clés : 


\section{Introduction}

The biophysics of neurons and their ionic channels are now understood in great details, although many questions remain [Hille(2001)]. Yet, simple neuron models such as the integrate-and-fire model [Lapicque(1907), Gerstner and Kistler(2002)] remain very popular in the computational neuroscience community, because they can be simulated very efficiently and, perhaps more importantly, because they are easier to understand and analyze. The drawback is that these simple models cannot account for the variety of electrophysiological behaviors of real neurons (see e.g. [Markram et al(2004)] for interneurons). Recently, several authors introduced two-variable spiking models [Izhikevich(2004), Brette and Gerstner(2005), Touboul(2008)] which, despite their simplicity, can reproduce a large number of electrophysiological signatures such as bursting or regular spiking. Different sets of parameter values correspond to different electrophysiological classes.

All these two-dimensional models are qualitatively similar, but we are especially interested in the adaptive exponential integrate-and-fire model (AdEx, [Brette and Gerstner(2005)]) because its parameters can be easily related to physiological quantities, and the model has been successfully fit to a biophysical model of a regular spiking pyramidal cell and to real recordings of pyramidal cells [Clopath et al(2007)Clopath, Jolivet, Rauch, Lüscher, and Gerstner, Jolivet et al(2008)Jolivet, Kobayashi, Rauch, Naud, Shinom This model is described by two variables, the membrane potential $V$ and an adaptation current $w$, whose dynamics are governed by the following differential equations:

$$
\left\{\begin{aligned}
C \frac{\mathrm{d} V}{\mathrm{~d} t}= & -g_{L}\left(V-E_{L}\right)+g_{L} \Delta_{T} \exp \left(\frac{V-V_{T}}{\Delta_{T}}\right) \\
& -w+I \\
\tau_{w} \frac{\mathrm{d} w}{\mathrm{~d} t}= & a\left(V-E_{L}\right)-w
\end{aligned}\right.
$$

When the membrane potential $V$ is high enough, the trajectory quickly diverges because of the exponential term. This divergence to infinity models the spike (the shape of the action potential is ignored, as in the standard integrate-and-fire model). For displaying or simulation purposes, spikes are usually cut to some finite value (e.g. $0 \mathrm{mV}$ ). When a spike occurs, the membrane potential is instantaneously reset to some value $V_{r}$ and the adaptation current is increased:

$$
\begin{cases}V & \rightarrow V_{r} \\ w & \rightarrow w+b\end{cases}
$$

Although the differential system is only two-dimensional, the reset makes the resulting dynamical hybrid system very rich.

The differential equations and the parameters have a physiological interpretation. The first equation is the membrane equation, which states that the capacitive current through the membrane ( $C$ is the membrane capacitance) is the sum of the injected current $I$ and of the ionic currents. The first term is the leak current ( $g_{L}$ is the leak conductance and $E_{L}$ is the leak reversal potential), the membrane time constant is $\tau_{m}=C / g_{L}$. The second (exponential) term approximates the sodium current, responsible for the generation of action potentials [Fourcaud-Trocme et al(2003)Fourcaud-Trocme, Hansel, van Vreeswijk, and B The approximation results from neglecting the inactivation of the sodium channel and assuming that 
activation is infinitely fast (which is reasonable). Because activation curves are typically Boltzmann functions [Angelino and Brenner(2007)], the approximated current is exponential near spike initiation. The voltage threshold $V_{T}$ is the maximum voltage that can be reached without generating a spike (without adaptation), and the slope factor $\Delta_{T}$ quantifies the sharpness of spikes. In the limit of zero slope factor, the model becomes an integrate-and-fire model with a fixed threshold $V_{T}$. Quantitatively, it is proportional to the slope constant $k$ in the activation function of the sodium current. The second variable $w$ is an adaptation current with time constant $\tau_{w}$, which includes both spike-triggered adaptation, through the reset $w \rightarrow w+b$, and subthreshold adaptation, through the coupling (variable a). It may model ionic channels (e.g. potassium) or a dendritic compartment. Quantitatively, the coupling variable $a$ can result from a linearization of the dynamics of a ionic channel, or from the axial conductance in the case of a dendritic compartment. We generally assume $a>0$ in this paper, although the analysis also applies for $a<0$ when $a$ is not too large.

The interaction of the differential equations with the reset results in a rich dynamical structure. There are 9 parameters plus the injected current $I$, but these can be reduced to 4 variables plus the current $I$ by changes of variables (e.g. setting $V_{T}$ as the reference potential, $\Delta_{T}$ as the voltage unit, $\tau_{m}$ as the time unit, etc.). Thus, the electrophysiological class of the model, defined loosely here as the set of qualitative behaviours for different values of $I$, is parameterized in a 4-dimensional space. In this paper, we will make this definition more precise by explaining different electrophysiological signatures in terms of dynamics of the model. Because we are dealing with a hybrid dynamical system, we shall study here two distinct dynamical aspects of the model: the subthreshold dynamics, defined by the differential equations (section 2), and the spiking dynamics, defined the sequence of resets (section 3). The former case was addressed by [Touboul(2008)] in a more general setting: we will review some of those results in the specific context of the adaptive integrate-and-fire model, and present new specific results, in particular about oscillations, attraction basins and rebound properties. In the latter case, we will see that the spike patterns of the model correspond to orbits under a Poincaré map, which we shall call the adaptation map $\Phi$. Interestingly, we find that this map can have chaotic dynamics under certain circumstances. Although we focus on this model for the reasons mentioned above, many results also apply when the membrane equation is replaced by a more general equation $d V / d t=F(v)-w+I$, where $F$ is a smooth convex function whose derivative is negative at $-\infty$ and infinite at $+\infty$ (in particular, Izhikevich model and the quartic model have these properties, see [Touboul(2008)]).

All simulations shown in this paper were done with the Brian software [Goodman and Brette(2008)]

The code is available on ModelDB at the following URL: http://senselab.med.yale.edu/ modeldb/ShowModel . asp?model=114242.

\section{Subthreshold dynamics}

\subsection{Rescaling}

The equations can be written in dimensionless units by expressing time in units of the membrane time constant $\tau_{m}=C / g_{L}$, voltage in units of the slope factor $\Delta_{T}$ and with reference potential $V_{T}$, and rewriting both the adaptation variable $w$ and the input current $I$ in voltage units. We obtain the 
following equivalent model:

$$
\left\{\begin{array}{l}
\frac{d \bar{V}}{d \bar{t}}=-\bar{V}+e^{\bar{V}}-\bar{w}+\bar{I} \\
\overline{\tau_{w}} \frac{d \bar{w}}{d \bar{t}}=\bar{a} \bar{V}-\bar{w}
\end{array}\right.
$$

and when a spike is triggered:

$$
\begin{cases}\bar{V} & \rightarrow \bar{V}_{r} \\ \bar{w} & \rightarrow \bar{w}+\bar{b}\end{cases}
$$

where

$$
\begin{cases}\overline{\tau_{w}} & :=\frac{\tau_{w}}{\tau_{m}}=\frac{g_{L} \tau_{w}}{C} \\ \bar{a} & :=\frac{a}{g_{L}} \\ \bar{I} & :=\frac{I}{g_{L} \Delta_{T}}+\left(1+\frac{a}{g_{L}}\right) \frac{E_{L}-V_{T}}{\Delta_{T}} \\ \bar{t} & :=\frac{t}{\tau_{m}} \\ \bar{b} & :=\frac{b}{g_{L} \Delta_{T}} \\ \overline{V_{r}} & :=\frac{V_{r}-V_{T}}{\Delta_{T}} \\ \bar{V}(\bar{t}) & :=\frac{V(t)-V_{T}}{\Delta_{T}} \\ \bar{w}(\bar{r}) & :=\frac{w(t)+a\left(E_{L}-V_{T}\right)}{g_{L} \Delta_{T}}\end{cases}
$$

It appears that the model has only four free parameters (plus the input current). In this section we will focus on the differential equations; we will turn to the sequence of resets in section 3 . Thus, only two parameters characterize the subthreshold dynamics: the ratio of time constants $\tau_{w} / \tau_{m}$ and the ratio of conductances $a / g_{L}$ (note: $a$ can be seen as the stationary adaptation conductance).

The rescaled model belongs to the class studied in [Touboul(2008)] with $F(v)=e^{v}-v$, i.e., $F$ is convex, three times continuously differentiable, has a negative derivative at $-\infty$ and an infinite derivative at $+\infty$. Therefore it has the same bifurcation structure, which we will develop here and relate to electrophysiological properties We also provide formulas for the excitability type, rheobase current, voltage threshold and the I-V curve. Besides, we give quantitative conditions for the occurence of oscillations, along with a formula for their frequency. Finally, we examine the rebound properties of the model, in relationship with the attraction basin of the stable fixed point.

\subsection{Excitability}

The dynamics in the phase plane $(V, w)$ are partly determined by the number and nature of fixed points, which are the intersections of the two nullclines (Fig. 1):

$$
\begin{aligned}
& w=-g_{L}\left(V-E_{L}\right)+g_{L} \Delta_{T} \exp \left(\frac{V-V_{T}}{\Delta_{T}}\right)+I \quad(V \text {-nullcline }) \\
& w=a\left(V-E_{L}\right) \quad(w \text {-nullcline })
\end{aligned}
$$

Because the membrane current (first equation) is a convex function of the membrane potential $V$, there can be no more than two fixed points. When the input current $I$ increases, the V-nullcline goes up and the number of fixed points goes from two to zero, while the trajectories go from resting

$\mathrm{RR} \mathrm{n}^{\circ} 6563$ 


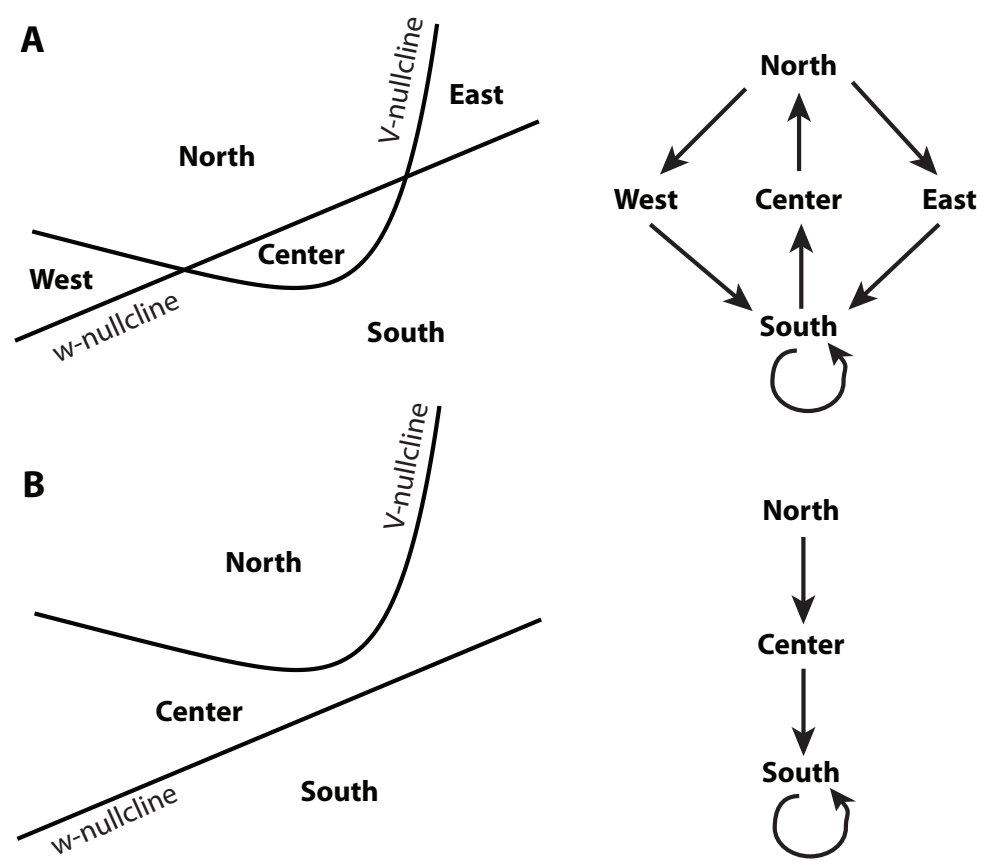

Figure 1: Nullclines of the dynamical system (horizontal axis: $V$; vertical axis: $w$ ). A. The nullclines intersect in two points, and divide the phase space into 5 regions. The potential $V$ increases below the $V$-nullcline, $w$ increases below the $w$-nullcline. The direction of the flow along each boundary gives the possible transitions between regions (right). Spiking can only occur in the South region. B. The nullclines do not intersect. All trajectories must enter the South region and spike. 
Type I (saddle-node)

A

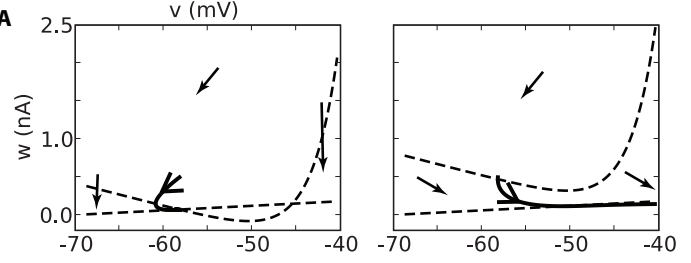

Type II (Andronov-Hopf)

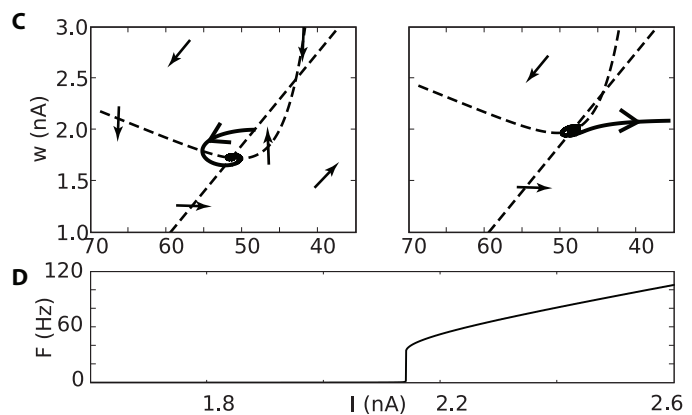

Figure 2: Excitability types. A,B. Type I: $\frac{a}{g_{L}}<\frac{\tau_{m}}{\tau_{w}}$ (here: $a=.2 g_{L}, \tau_{m}=3 \tau_{w}$ ). When $I$ is increased, the resting point disappears through a saddle-node bifurcation: the two fixed points merge and disappear. The current-frequency curve is continuous (B). C,D. Type II: $\frac{a}{g_{L}}>\frac{\tau_{m}}{\tau_{w}}$ (here: $a=3 g_{L}$, $\left.\tau_{m}=.5 \tau_{w}\right)$. When $I$ is increased, the resting point becomes unstable through an Andronov-Hopf bifurcation: the stable fixed point becomes unstable. The current-frequency curve is discontinuous, there is a non-zero minimum frequency (D).

to spiking. The excitability properties of the model depend on how the transition to spiking occurs, that is, on the bifurcation structure.

\subsubsection{Excitability types}

When $I$ is very negative, there are two fixed points, one of which is stable (the resting potential). It appears that, when increasing $I$, two different situations can occur depending on the quantity $\frac{a \tau_{w}}{C}=\frac{a}{g_{L}} \frac{\tau_{w}}{\tau_{m}}$ (ratio of conductances times ratio of time constants).

If $\frac{a}{g_{L}}<\frac{\tau_{m}}{\tau_{w}}$, then the system undergoes a saddle-node bifurcation when $I$ is increased, i.e., the stable and unstable fixed points merge and disappear. This fact implies that the model has type I excitability, that is, the current-frequency curve is continuous (Fig. 2). Indeed, when the fixed points disappear, the vector field is almost null around the former fixed point (the ghost of the fixed point). Since the vector field can be arbitrarily small close to the bifurcation, the trajectory can be trapped for an arbitrarily long time in the ghost of the fixed point, so that the firing rate can be arbitrary small when $I$ is close to the bifurcation point (threshold). This property also explains the phenomenon of spike latency.

If $\frac{a}{g_{L}}>\frac{\tau_{m}}{\tau_{w}}$, then the system undergoes an Andronov-Hopf bifurcation before the saddle-node one, meaning that the stable fixed point first becomes unstable before merging with the other fixed point. This fact implies that the model has type II excitability, that is, the current-frequency curve is discontinuous at threshold, the firing rate suddenly jumps from zero to a finite value when the bifurcation point is crossed (Fig. 2).

The bifurcation for the limit case $\frac{a}{g_{L}}=\frac{\tau_{m}}{\tau_{w}}$ is called a Bogdanov-Takens bifurcation. It has codimension two, i.e. it appears when simultaneously varying the two parameters $\bar{a}$ and $\bar{I}$ of the rescaled 
model. At this point, the family of unstable periodic orbits generated around the Andronov-Hopf bifurcation collides with the saddle fixed point and disappears via a saddle-homoclinic bifurcation. There is no other bifurcation in this model (as well as in Izhikevich model [Izhikevich(2004)]). Other similar models such as the quartic model may also undergo a Bautin bifurcation, associated with stable oscillations (see [Touboul(2008)]).

The fixed points can be calculated using the Lambert function $W$, which is the inverse of $x \mapsto x e^{x}$ :

$$
\left\{\begin{array}{l}
V_{-}:=E_{L}+\frac{I}{g_{L}+a}-\Delta_{T} W_{0}\left(-\frac{1}{1+a / g_{L}} e^{\frac{I}{\Delta_{T}\left(g_{L}+a\right)}+\frac{E_{L}-V_{T}}{\Delta_{T}}}\right) \\
V_{+}:=E_{L}+\frac{I}{g_{L}+a}-\Delta_{T} W_{-1}\left(-\frac{1}{1+a / g_{L}} e^{\frac{I}{\Delta_{T}\left(g_{L}+a\right)}+\frac{E_{L}-V_{T}}{\Delta_{T}}}\right)
\end{array}\right.
$$

where $W_{0}$ is the principal branch of the Lambert function and $W_{-1}$ the real branch of the Lambert function such that $W_{-1}(x) \leq-1$, defined for $-e^{-1} \leq x<1$ (indeed since $x \mapsto x e^{x}$ is not injective, the Lambert function is multivalued).

The fixed point $V_{+}$is always a saddle fixed point (hence unstable), i.e. its Jacobian matrix has an eigenvalue with positive real part and an eigenvalue with negative real part. The fixed point $V_{-}$is stable if the model is type I, otherwise it depends on the current $I$, as we discuss below.

\subsubsection{Rheobase current}

The rheobase current is the minimum constant current required to elicit a spike, i.e., the first point when the stable fixed point becomes unstable, which depends on the excitability type.

For type I $\left(\frac{a}{g_{L}} \frac{\tau_{w}}{\tau_{m}}<1\right)$, it corresponds to the saddle-node bifurcation point:

$$
I_{\mathrm{rh}}^{I}=\left(g_{L}+a\right)\left[V_{T}-E_{L}-\Delta_{T}+\Delta_{T} \log \left(1+\frac{a}{g_{L}}\right)\right]
$$

which is obtained by calculating the intersection of the nullclines when these are tangent.

For type II $\left(\frac{a}{g_{L}} \frac{\tau_{w}}{\tau_{m}}>1\right)$, it corresponds to the Andronov-Hopf bifurcation point:

$$
\begin{aligned}
& I_{\mathrm{rh}}^{I I}=\left(g_{L}+a\right)\left[V_{T}-E_{L}-\Delta_{T}+\Delta_{T} \log \left(1+\frac{\tau_{m}}{\tau_{w}}\right)\right] \\
& +\Delta_{T} g_{L}\left(\frac{a}{g_{L}}-\frac{\tau_{m}}{\tau_{w}}\right)
\end{aligned}
$$

It is important to note that the saddle-node bifurcation also occurs in the type II case at the point $I_{S N}=I_{r h}^{I}\left(>I_{\mathrm{rh}}^{I I}\right.$; for type II we use $I_{S N}$ instead of $I_{r h}^{I}$ to avoid ambiguities).

\subsubsection{Voltage threshold for slow inputs}

For a parameterized input $I_{a}(t)$, the threshold is the minimum value of the parameter $a$ for which a spike is elicited. For example, the rheobase current is the threshold constant current. However, the notion of a spike threshold for neurons is often described as a voltage threshold, although the voltage is not a stimulation parameter (thus, it implicitly refers to an integrate-and-

fire model). It is nevertheless possible to define a meaningful voltage threshold for the case of 
constant current inputs as follows: the voltage threshold is the maximum stationary voltage $V$ for subthreshold constant current inputs $\left(I \leq I_{\mathrm{rh}}\right)$. For the exponential integrate-and-fire model [Fourcaud-Trocme et al(2003)Fourcaud-Trocme, Hansel, van Vreeswijk, and Brunel], this is simply $V_{T}$. For the present model, it corresponds to the voltage $V_{-}$at the first bifurcation point, when the stable fixed point becomes unstable.

Not surprisingly, its value depends on the excitability type. For type I excitability $\left(a / g_{L}<\right.$ $\tau_{m} / \tau_{w}$ ), the voltage threshold is

$$
V_{\text {threshold }}^{\text {slow }}=V_{T}+\Delta_{T} \log \left(1+a / g_{L}\right)
$$

For type II excitability $\left(a / g_{L}<\tau_{m} / \tau_{w}\right)$, the voltage threshold is

$$
V_{\text {threshold }}^{\text {slow }}=V_{T}+\Delta_{T} \log \left(1+\tau_{m} / \tau_{w}\right)
$$

Interestingly, the threshold for type I excitability depends on the ratio of conductances, while the threshold for type I excitability depends on the ratio of time constants.

\subsubsection{Voltage threshold for fast inputs}

For short current pulses ( $I=q \delta(t)$, where $q$ is the total charge and $\delta(t)$ is the Dirac function), the voltage threshold is different, but the same definition may be used: it is the maximum voltage $V$ that can be reached without triggering a spike. Injecting short current pulses amounts to instantaneously changing the membrane potential $V$, i.e., in the phase space $(V, w)$, to moving along an horizontal line. If, by doing so, the point $(V, w)$ exits the attraction basin of the stable fixed point, then a spike is triggered. Therefore, the threshold is a curve in the phase space, defined as the boundary of the attraction basin of the stable fixed point (for which we have unfortunately no analytical expression, although it can be computed numerically). Therefore the model displays threshold variability: the voltage threshold depends on the value of the adaptation variable $w$, i.e., on the previous inputs. The boundary of the attraction basin of the stable fixed point is either the stable manifold of the saddle fixed point(separatrix) or a limit cycle. We examine this issue in section 2.6 and in appendix C.

\subsection{I-V curve}

The I-V curve of a neuron is the relationship between the opposite of the (constant) injected current and the stationary membrane potential (it may also be defined for non-constant input currents, see e.g. [Badel et al(2008)Badel, Lefort, Brette, Petersen, Gerstner, and Richardson]). Experimentally, this curve can be measured with a voltage-clamp recording. We obtain a simple expression by calculating $I$ at the intersection of the nullclines:

$$
I(V)=\left(a+g_{L}\right)\left(V-E_{L}\right)-g_{L} \Delta_{T} \exp \left(\frac{V-V_{T}}{\Delta_{T}}\right)
$$

Thus, far from threshold, the $I-V$ curve is linear and its slope is the leak conductance plus the adaptation conductance. 


\subsection{Oscillations}

Because of the coupling between the two variables $V$ and $w$, there can be oscillations near the resting potential, more precisely, damped oscillations (self-sustained oscillations are not possible in this model, nor in Izhikevich model, as is shown in [Touboul(2008)]). Oscillations occur when the eigenvalues associated with the stable fixed point are complex; when they are real, solutions converge (locally) exponentially to the stable fixed point.

Because of the nature of the bifurcations, near the rheobase current (section 2.2.2), the model is non-oscillating if it has excitability type I $\left(a / g_{L}<\tau_{m} / \tau_{w}\right)$ and oscillating if it has type II. Far from threshold, these properties can change. In this section we give explicit expressions for the parameter zones corresponding to both regimes; details of the calculations are detailed in appendix A for the rescaled model (3).

The parameter zones depend on the excitability types, the ratio $\tau_{w} / \tau_{m}$ and the following condition:

$$
\frac{a}{g_{L}}<\frac{\tau_{m}}{4 \tau_{w}}\left(1-\frac{\tau_{w}}{\tau_{m}}\right)^{2}
$$

These results are summarized in Fig. 3.

\subsubsection{Oscillations for type I}

Three cases appear:

- If inequality (9) is false, then the model oscillates when $I<I_{+}$, where the formula for $I_{+}$is given in Appendix A. In practice, we observe that $I_{+}$is very close to the rheobase current, so that the model almost always oscillates below threshold.

- If inequality (9) is true and $\tau_{m}>\tau_{w}$, then the model never oscillates near the fixed point.

- If inequality (9) is true and $\tau_{m}<\tau_{w}$, then the model oscillates when $I_{-}<I<I_{+}$, where the formula for $I_{-}$is given in Appendix A.

\subsubsection{Oscillations for type II}

Two cases appear:

- If inequality (9) is false, then the model always oscillates near the fixed point, for any subthreshold input current $I$.

- If inequality (9) is true, then the model oscillates only when $I>I_{-}$.

We call the occurrence of oscillations the resonator regime and their absence the integrator regime (see 2.5.1). The model is called a resonator when it is always (for all $I$ ) or almost always (for $I<I_{+}$) in the resonator regime, i.e., when inequality (9) is false; it is called an integrator when it never oscillates, i.e., when $\tau_{m}>\tau_{w}$ and inequality (9) is true; it is said to be in a mixed mode when it oscillates only above some value $I_{-}$(see Fig. 3). 


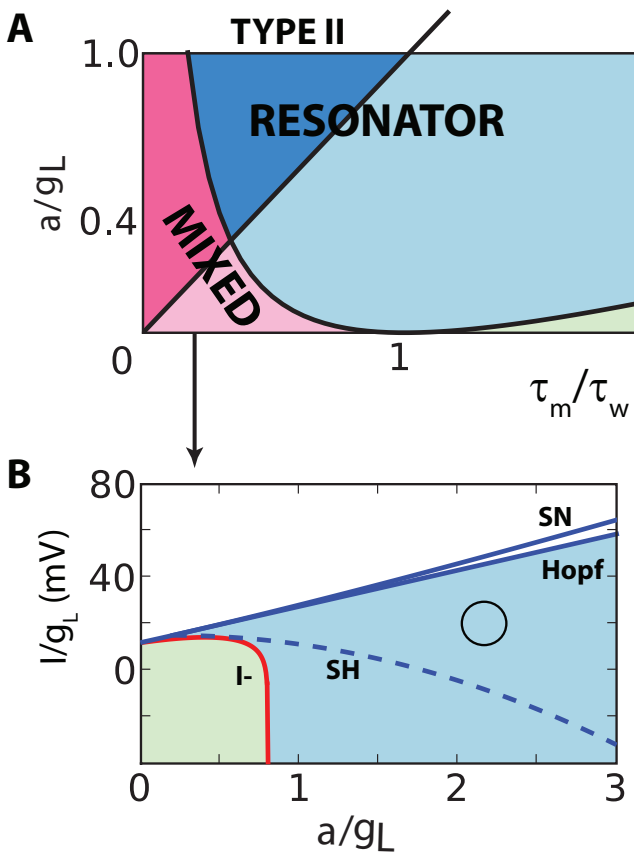

\section{TYPE I}
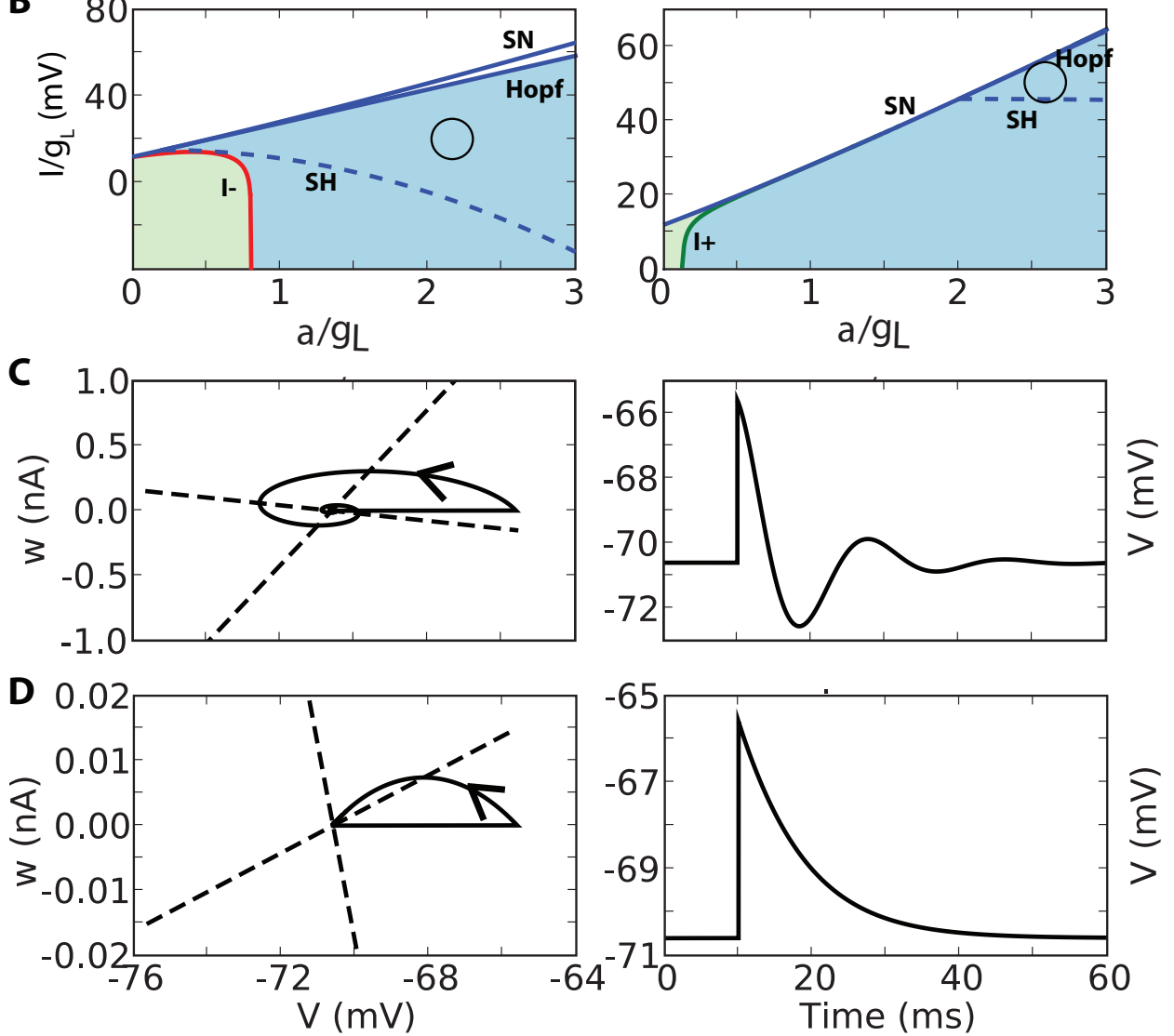

Figure 3: Oscillations. A. Behavior of the model as a function of $a / g_{L}$ and $\tau_{m} / \tau_{w}$. Light (dark) colors indicate type I (type II) excitability. Blue: resonator mode (oscillations for any or almost any $I$ ). Green: integrator mode (oscillations for any $I$ ). Pink: mixed mode (resonator if $I$ is large enough, otherwise integrator). B. Behavior of the model as a function of $a / g_{L}$ and $I / g_{L}$ for $\tau_{m}=.2 \tau_{w}$ (left) and $\tau_{m}=2 \tau_{w}$ (right). White: spiking; blue: oscillations; green: no oscillation. Spiking occurs when $I$ is above the saddle-node curve (SN) in the type I regime, and above the Hopf curve (Hopf) in the type II regime. A repulsive limit cycle (circle) exists when $I$ is above the saddle-homoclinic curve (SH; only for type II). Oscillations occur when $I_{-}<I<I_{+}$(on the left, $I_{+} \geq I_{S N}$; on the right, $\left.I_{-}={ }^{\circ}{ }^{\circ} \infty 6\right)$. C,D. Response of the system to a short current pulse (Dirac) near the resting point, in the resonator regime $\left(\mathrm{C} ; a=10 g_{L}, \tau_{m}=\tau_{w}\right)$ and in the integrator regime $\left(\mathrm{D} ; a=.1 g_{L}, \tau_{m}=2 \tau_{w}\right)$. Left: response in the phase space $(V, w)$; right: voltage response in time. 


\subsubsection{Frequency of oscillations}

When the model oscillates, the frequency of the oscillations is:

$$
F=\frac{\omega}{2 \pi}=\frac{2 a}{\pi g_{L} \tau_{w}}-\frac{2}{\pi \tau_{m}}\left(e^{\frac{V_{-}-V_{T}}{\Delta_{T}}}-1+\frac{\tau_{m}}{\tau_{w}}\right)^{2},
$$

which can be approximated far from threshold $\left(V_{-} \ll V_{T}\right)$ as follows:

$$
F=\frac{\omega}{2 \pi} \approx \frac{2 a}{\pi g_{L} \tau_{w}}-\frac{2}{\pi \tau_{m}}\left(1-\frac{\tau_{m}}{\tau_{w}}\right)^{2} .
$$

\subsection{Input integration}

The way the model integrates its inputs derives from the results above.

\subsubsection{Resonator vs. integrator}

On the temporal axis, the integration mode can be defined locally (for a small input $I(t)$ ) as

$$
V(t)=V_{0}+(K \star I)(t)
$$

where the kernel $K$ is the linear impulse response of the model around $V_{0}$, and $K \star I$ is a convolution. This impulse response is determined by the eigenvalues of the stable fixed point. When these are complex, the kernel $K$ oscillates (with an exponential decay), as discussed in section 2.4 (see Fig. $3 \mathrm{C}$ ). In that case the model acts as a resonator: two inputs are most efficient when separated by the characteristic oscillation period of the model (given by eq. 10). The membrane time constant is $-1 / \lambda$, where $\lambda$ is the real part of the eigenvalues. Far from threshold $\left(V<<V_{T}\right)$, we find the following time constant (see Appendix A):

$$
\tau=2 \frac{\tau_{m} \tau_{w}}{\tau_{m}+\tau_{w}}
$$

When the eigenvalues are real, the kernel $K$ is a sum of two exponential functions, and the model acts as an integrator. In that case there are two time constants, given by the real part of the eigenvalues. It is interesting to note that there is a parameter region where both integration modes can exist, depending on the (stationary) input current $I$ : oscillations arise only when the model is sufficiently depolarized $\left(I>I_{-}\right)$.

\subsubsection{Adaptation}

There are two sorts of adaptation in the model: threshold adaptation and voltage adaptation. The former one comes from the orientation of the separatrix in the $(V, w)$ plane, as we discussed in section 2.2.4. The latter one derives from the fact that in the integrator mode (no oscillation), the model kernel $K$ is a sum of two exponential functions. If the slower one is negative, then the response 
to a step shows an overshoot (as in Fig. 4D for a negative current step), which is a form of adaptation (the voltage response is initially strong, then decays). That overshoot can be seen when there is no oscillation and $\tau_{m}<\tau_{w}$ (see Appendix B), i.e., in the mixed mode shown in pink in Fig. 3, when the input current is low $\left(I<I_{-}\right)$.

\subsection{The attraction basin of the stable fixed point}

\subsubsection{Limit cycle}

The existence of a repulsive limit cycle arises for type II excitability from the Andronov-Hopf bifurcation. The saddle-node and Andronov-Hopf bifurcations collide via a Bogdanov-Takens bifurcation. In the neighborhood of this bifurcation, the family of limit cycles disappears via a saddlehomoclinic bifurcation. The normal form of the Bogdanov-Takens bifurcation gives us a local approximation of this saddle-homoclinic bifucation curve around the point in parameter space given by (12) (see [Touboul(2008)]), and the full saddle-homoclinic curve can be computed numerically using a continuation algorithm. The current $I$ above which a limit cycle exists is locally approximated at the second order by the following expression:

$$
I_{\text {cycle }}=I_{B T}-\frac{12}{25} \frac{\Delta_{T} \tau_{w}^{2}}{C\left(\tau_{m}+\tau_{w}\right)}\left(a-\frac{C}{\tau_{w}}\right)^{2}+o\left(a_{1}^{2}\right)
$$

for $a>\frac{C}{\tau_{w}}$, where $I_{B T}$ is the rheobase current at the Bogdanov-Takens bifurcation:

$$
I_{B T}=\left(g_{L}+\frac{C}{\tau_{w}}\right)\left[V_{T}-E_{L}-\Delta_{T}+\Delta_{T} \log \left(1+\frac{C}{g_{L} \tau_{w}}\right)\right]
$$

Below the threshold current $I_{\text {cycle, }}$, there is no limit cycle (see next section). Above the $I_{\text {cycle, }}$ there is a repulsive limit cycle, circling anti-clockwise around the stable fixed point (see Fig. 3B and 4A); the saddle fixed point is outside that cycle. Interestingly, it appears that one can exit the attraction basin of the stable fixed point (and thus generate a spike) not only by increasing $V$, but also by decreasing $V$ or $w$ (or increasing $w$ ). This phenomenon is sometimes called rebound, and we discuss it further in section 2.7 .

\subsubsection{Separatrix}

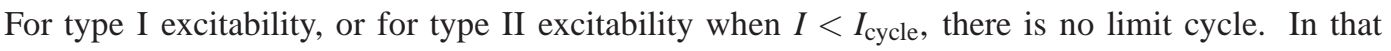
case the stable manifold of the saddle fixed point is an unbounded separatrix, i.e., it delimits the attraction basin of the stable fixed point. From the position of the nullclines, it appears that the stable manifold must cross the saddle fixed point from above both nullclines (North) to below both nullclines (South). It follows that the side above the nullclines is the graph of an increasing function of $V$ (see Fig. 4). As for the other part of the manifold, several cases can occur: it may cross the W-nullcline, both nullclines or none. One can show (appendix C) that if condition (9) is false (section 2.4 ), then both nullclines are crossed, and if $\tau_{m}<\tau_{w}$, then at least the w-nullcline is crossed. These conditions cover all parameter regions except the zone where the model is always an integrator (no 

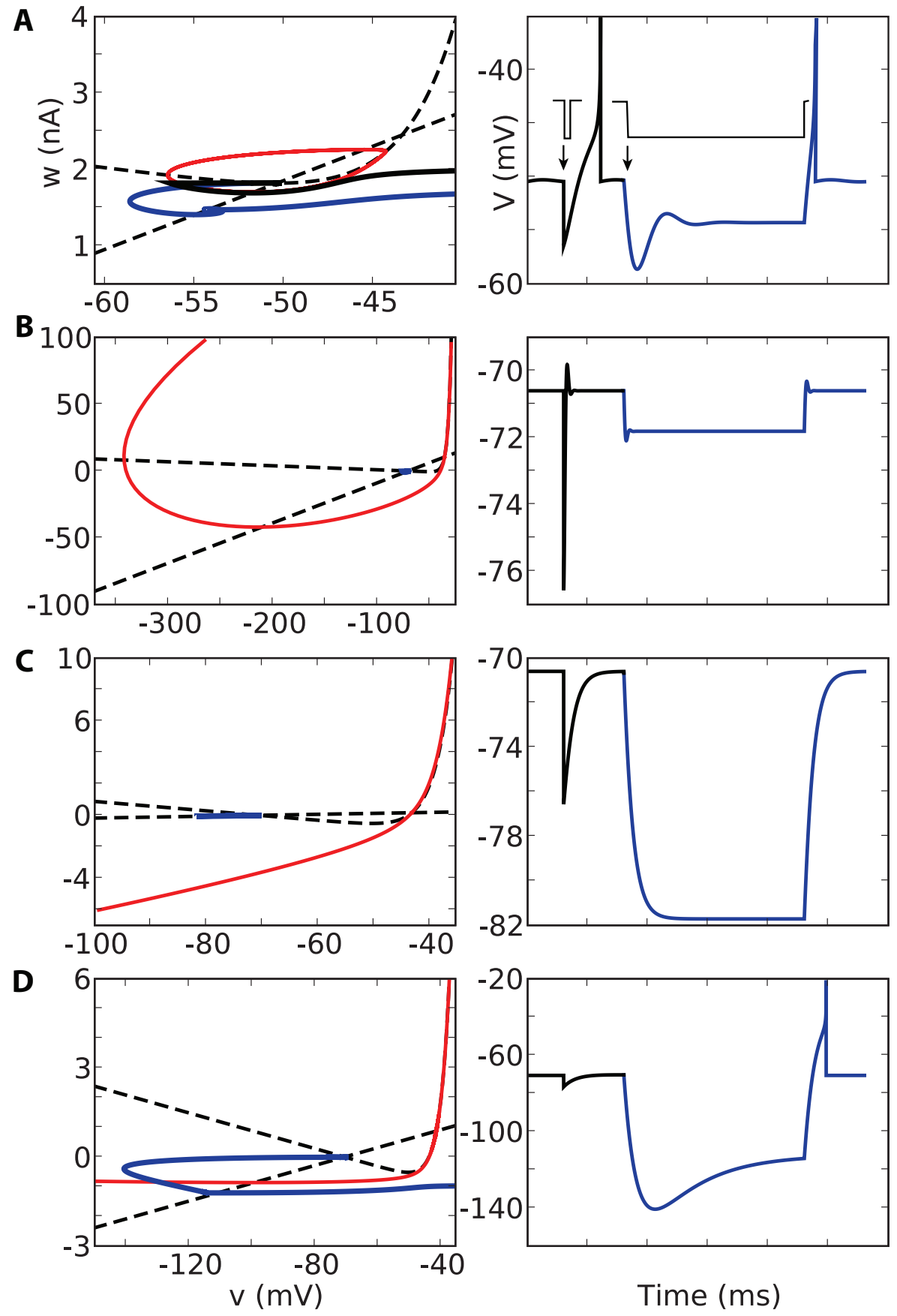

Figure 4: The attraction basin of the stable fixed point and rebound properties. Left column: the dashed lines represent the nullclines, each panel corresponds to a different set of parameter values; the red line delimits the attraction basin of the stable fixed point; the black line is the trajectory of the model in response to a short negative current pulse, while the blue line is the trajectory in response to a long negative current step. Right column: voltage response of the model to the a short pulse (bIREK) and to a long step (blue). A. Type II resonator ( $\left.a=3 g_{L}, \tau_{w}=2 \tau_{m}\right)$ close to the rheobase current. A repulsive limit cycle appears. Trajectories can escape the attraction basin and spike with fast or slow hyperpolarization. B. Type I resonator $\left(a=10 g_{L}, \tau_{m}=12 \tau_{w}\right)$. The separatrix crosses both nullclines (for both branches, $V$ and $w$ go to $+\infty$ ). In theory trajectories can escape the attraction basin with hyperpolarization, but one would need to reach unrealistically low voltages $(<-200$ $\mathrm{mV})$. C. Integrator $\left(a=.2 g_{L}, \tau_{m}=3 \tau_{w}\right)$. The separatrix does not cross the nullclines. No rebound is possible. D. Type II mixed mode $\left(a=g_{L}, \tau_{w}=10 \tau_{m}\right)$. The separatrix crosses the w-nullcline. Rebound is possible with long hyperpolarization (short hyperpolarization can also induce rebounds, but with unrealistically low voltages). 
oscillations); in particular, it includes the type II excitability zone. The position of the separatrix has important implications for the rebound property (section 2.7).

\subsection{Rebound}

The term rebound refers to the property that a spike can be triggered by hyperpolarizing the membrane. This can be done either by sending a short negative current pulse, which amounts to moving the state vector $(V, w)$ horizontally to the left, or by slowly hyperpolarizing the membrane with a long negative current step (or ramp) and releasing it, which amounts to moving the state vector along the w-nullcline.

For type I excitability, there is no limit cycle and there is an unbounded separatrix. If $\tau_{m}<\tau_{w}$ or if condition (9) is false, then the separatrix crosses the w-nullcline. It follows that both types of rebounds are possible. Otherwise the model is in the integrator regime, and the the separatrix may not cross the w-nullcline. In that case it is only possible to trigger a spike by increasing the voltage: there is no rebound.

For type II excitability, there is either a repulsive limit cycle which circles the stable fixed point when the input current is close enough to the rheobase current $\left(I>I_{\text {cycle }}\right)$, or the separatrix crosses both the w-nullcline and the v-nullcline. In both cases, it is possible to exit the attraction basin of the stable fixed point and thus trigger a spike by changing any variable in any direction. Therefore, both types of rebound are possible. Note that with short current pulses, a more negative voltage must be reached in order to trigger a spike.

\subsection{After-potential}

After a spike, the state vector resets to a certain point in the state space. The subsequent trajectory is determined by this initial state. We will discuss the spike sequences in more details in section 3 , but here we simply note that if the state vector is reset above the V-nullcline, then the membrane potential $V$ will first decrease then increase (broad after-potential); if the state vector is reset below the V-nullcline, $V$ will increase (sharp after-potential).

\section{Spike patterns}

In the previous section, we analyzed the subthreshold dynamics of the model and found a rich structure, with the two parameters $a / g_{L}$ and $\tau_{m} / \tau_{w}$ controlling excitability, oscillations and rebound properties. Here we turn to the patterns of spikes, such as regular spiking, tonic/phasic bursting or irregular spiking, and explain them in terms of dynamics. Compared to the previous section, two additional parameters play an important role: the reset value $V_{r}$ and the spike-triggered adaptation parameter $b$.

To study the spike sequences, we introduce a Poincare map which transforms the continuous time dynamics of the system into the discrete time dynamics of that map. 

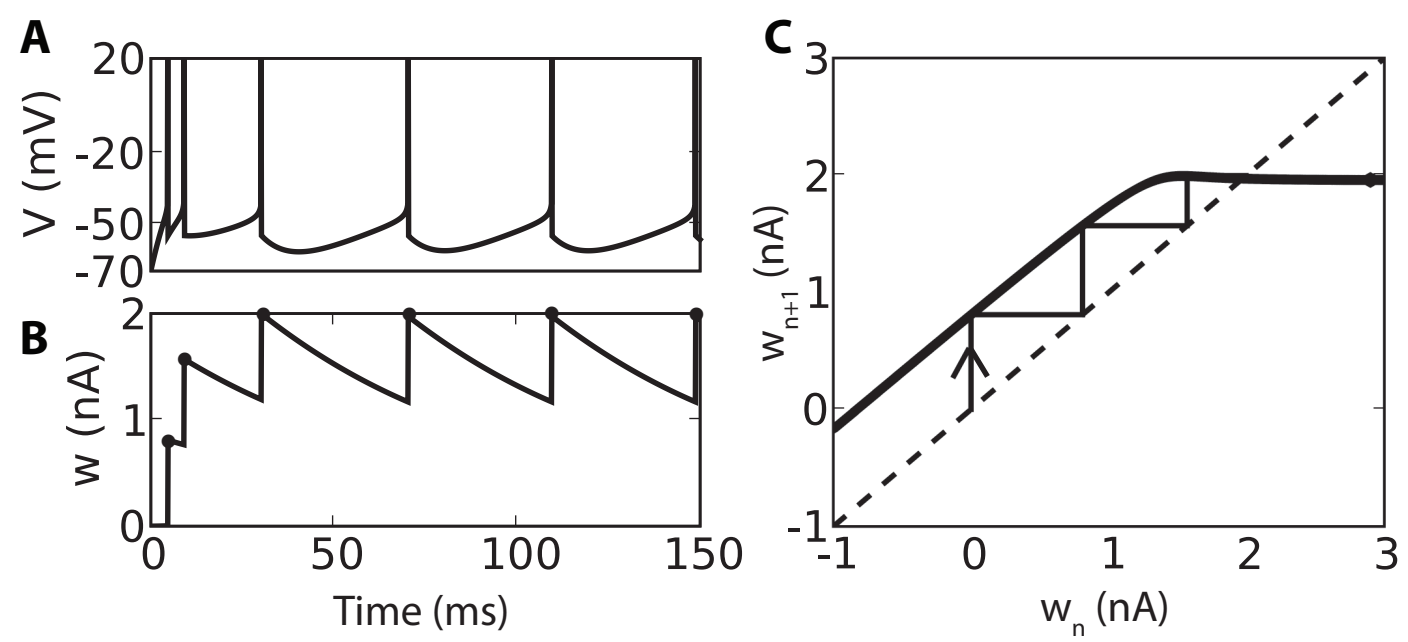

Figure 5: The adaptation map. A, B. Response of a type I model to a suprathreshold constant input (A: membrane potential $V$; B: adaptation variable $w$ ). The value of $w$ after each spike defines a sequence $\left(w_{n}\right)$. C. The adaptation map $\Phi$ maps the value of the adaptation variable from one spike to the next. The sequence $\left(w_{n}\right)$ is the orbit of $w_{0}$ under $\Phi$.

\subsection{The adaptation map}

After a spike, the potential $V$ is always reset to the same value $V_{r}$, therefore the trajectory is entirely determined by the value of the adaptation variable $w$ at spike time: the sequence of values $\left(w_{n}\right), w_{n}=$ $t_{n}\left(t_{n}=\right.$ time of spike number $\left.n\right)$ uniquely determines the trajectory after the first spike. Therefore, it is useful to introduce the function $\Phi$ mapping $w_{n}$ to $w_{n+1}$, which we call the adaptation map. Let us define $\mathscr{D}$ as the domain of the adaptation variable $w$ such that the solution of (1) with initial condition $\left(V_{r}, w\right)$ spikes (blows up in finite time). Then the adaptation map $\Phi$ is

$$
\Phi:\left\{\begin{array}{l}
\mathscr{D} \mapsto \mathbb{R} \\
w_{0} \mapsto w_{\infty}+b
\end{array}\right.
$$

where $w_{\infty}$ is the value of $w$ at divergence time (spike time) for the trajectory starting from $\left(V_{r}, w_{0}\right)$, as illustrated in Fig. 5. The sequence $\left(w_{n}\right)$ is the orbit of $w_{0}$ under $\Phi$, as shown in Fig. 5C. Note that this sequence may be finite if for some $n, w_{n} \notin \mathscr{D}$. The property that the sequence is infinite (resp. finite) is called tonic spiking (resp. phasic spiking). The spike patterns are determined by the dynamical properties of $\Phi$ (fixed points, periodic orbits, etc.), as we show in next section. First, we examine the spiking domain $\mathscr{D}$.

When there is no stable fixed point, i.e., when $I$ is above the rheobase current (section 2.2.2), either $I_{\mathrm{rh}}^{I}$ or $I_{\mathrm{rh}}^{I I}$ depending on the excitability type, then any trajectory spikes: $\mathscr{D}=\mathbb{R}$. When there is a stable fixed point, all trajectories starting inside the attraction basin of that fixed point will not 

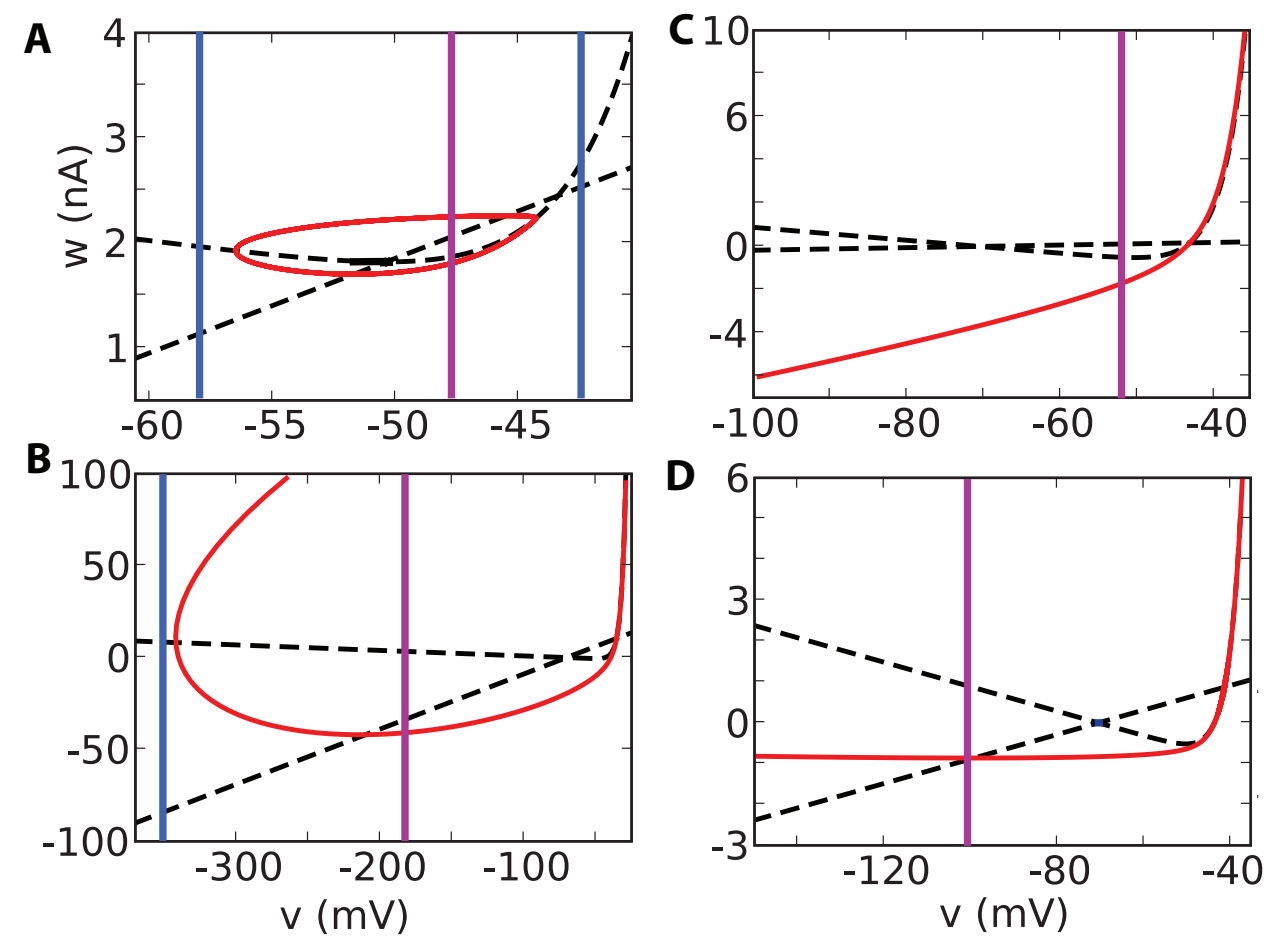

Figure 6: The spiking domain $\mathscr{D}$ for the same cases as in Fig. 4, when the nullclines (dashed lines) intersect. The attraction basin of the stable fixed point is bounded by the red curve. The blue and purple vertical lines indicate the reset line $V=V_{r}$. When that line is outside the attraction basin (blue), then $\mathscr{D}=\mathbb{R}$ and the model is bistable (tonic/resting). When the line intersects the attraction basin (purple), then $\mathscr{D}$ is an interval or the union of two intervals. In that case, the model is generally phasic (C,D) but may be bistable $(\mathrm{A}, \mathrm{B})$. In practice, with realistic values of $b$ (spiketriggered adaptation), bistability essentially occurs when there is a limit cycle (A). 
spike. The spiking domain $\mathscr{D}$ is then the complementary of the intersection of the reset line $V=V_{r}$ with the attraction basin of the stable fixed point (up to a projection onto the $w$ axis), as shown in Fig. 6. We previously found (2.6) that the attraction basin of the stable fixed point is either a limit cycle or the stable manifold of the saddle fixed point. In the latter case, it may have a minimum voltage (resonator) or not (integrator or mixed). Fig. 6 shows how these different cases determine the spiking domain $\mathscr{D}$. We summarize these findings below, and describe the adaptation map $\Phi$.

We first define two special values $w^{*}$ and $w^{* *}$ as follows: the reset line $V=V_{r}$ intersects the V-nullcline and w-nullcline at the points $\left(V_{r}, w^{*}\right)$ and $\left(V_{r}, w^{* *}\right)$, respectively, where

$$
\begin{cases}w^{*} & =-g_{L}\left(V_{r}-E_{L}\right)+g_{L} \Delta_{T} \exp \left(\frac{V_{r}-V_{T}}{\Delta_{T}}\right)+I \\ w^{* *} & =a\left(V_{r}-E_{L}\right)\end{cases}
$$

Nearby spiking trajectories starting on the reset line $V=V_{r}$ above $w^{*}$ (i.e., above the V-nullcline) may spike only after half a turn (since $V$ initially decreases), or possibly an odd number of halfturns, which implies that the vertical order of the trajectories is reversed at spike time: $\Phi$ is locally decreasing above $w^{*}$. Spiking trajectories starting below $w^{*}$ spike either directly or after an even number of half-turns, so that $\Phi$ is locally increasing below $w^{*}$. It follows that the sequences $\left(w_{n}\right)$ are bounded.

We now describe the map $\Phi$ and the spiking domain $\mathscr{D}$ for the two excitability types, depending on the input current $I$.

1. Type I:

(a) (subthreshold) if $I<I_{r h}^{I}$, then there is a stable fixed point and no limit cycle (see section 2.6). If the separatrix has no lower bound (typically: integrator or mixed regime), then the domain $\mathscr{D}$ is an interval $\left(-\infty, w_{\max }\right)$ where $w_{\max }$ is the value of the adaptation variable on the separatrix for $V=V_{r}$. The map $\Phi$ is continuous on that set. We note that if $V_{-}<V_{r}<V_{+}$, then there can only be phasing spiking: indeed, $w_{n+1}>w_{n}+b$ for all $n$, therefore at some point the orbit exits $\mathscr{D}$.

When the separatrix has a lower voltage bound $V_{\min }$ (typically: resonator), then there are two cases. If $V_{r}<V_{\min }$, then $\mathscr{D}=\mathbb{R}$ and $\Phi$ has the same properties as in case $1 \mathrm{~b}$. If $V_{r}>$ $V_{\min }$, then $\mathscr{D}=\left(-\infty, w_{\min }\right) \cup\left(w_{\max },+\infty\right)$. Besides, $\Phi\left(\left(w_{\max },+\infty\right)\right) \subset \Phi\left(\left(-\infty, w_{\min }\right)\right)$.

(b) (suprathreshold) if $I>I_{r h}^{I}$, all trajectories spike. Therefore, $\mathscr{D}=\mathbb{R}$. The adaptation map is concave for $w<w^{*}$, regular, has a unique fixed point and an a horizontal asymptote when $w \rightarrow+\infty$.

2. Type II:

(a) (subthreshold) if $I<I_{\text {cycle }}$, then there is a stable fixed point and no limit cycle, so that the situation is similar to case $1 \mathrm{~b}$.

(b) (subthreshold) if $I_{\text {cycle }}<I<I_{r h}^{I I}$, then there is a stable fixed point and a repulsive limit cycle bounding the attraction basin of the stable fixed point. Let $V_{\max }$ and $V_{\min }$ be the two extremal voltage values of the limit cycle. For $V_{r}<V_{\min }$ or $V_{r}>V_{\max }, \mathscr{D}=\mathbb{R}$ and $\Phi$ has the same properties as in case $1 b$. 
(c) (suprathreshold) if $I_{r h}^{I I}<I<I_{S N}$, then there are two unstable fixed points and no limit cycle, hence all trajectories spike. Therefore $\mathscr{D}=\mathbb{R}$. When $V_{r} \in\left(V_{-}, V_{+}\right)$, the adaptation map is discontinuous at some point $w_{\max }<w^{*}$, and $\Phi\left(w_{\max }\right)<\Phi\left(w_{\max }^{-}\right)$(when trajectories start circling around the fixed point). Thus $\Phi$ is locally but not globally increasing on $\left(-\infty, w^{*}\right)$. The map $\Phi$ also has a horizontal asymptote when $w \rightarrow+\infty$.

(d) (suprathreshold) if $I>I_{S N}$, then $\mathscr{D}=\mathbb{R}$ and $\Phi$ has the same properties as in case 1b (type I).

Tonic spiking occurs for any initial $w_{0}$ if $\mathscr{D}=\mathbb{R}$ (in particular, in the suprathreshold regime). In other cases, spiking is generally phasic but there can be tonic spiking if the set $\bigcap_{n=0}^{\infty} \Phi^{n}(\mathscr{D})$ is not empty. When it occurs, the model is bistable.

The sequence $\left(w_{n}\right)_{n \geq 0}$ of values of the adaptation variable at spike times is the orbit of $w_{0}$ under $\Phi: w_{n}=\Phi^{n}\left(w_{0}\right)$. Since there is a mapping from $w$ to the interspike interval, the properties of $\Phi$ determine the spike patterns. In the following, we examine the relationship between the adaptation map $\Phi$ and the spike patterns.

\subsection{Tonic Spiking}

\subsubsection{Regular Spiking}

Regular spiking means that interspike intervals are regular, possibly after a transient period of shorter intervals. For the adaptation variable, it means that the sequence $\left(w_{n}\right)$ converges, i.e., $\Phi$ has a stable fixed point. This situation is shown in Fig. 5. For low initial values of the adaptation variable, $\Phi$ is increasing and $\Phi(w)>w$, so that the sequence $\left(w_{n}\right)$ is increasing, implying that the duration of interspike intervals decreases (this implication is true for $w<w^{*}$, i.e., before the maximum of $\Phi$ ).

The shape of after-potentials (broad or sharp) depends, as we previously saw, on whether $\left(V_{r}, w\right)$ is above or below the V-nullcline, i.e., whether $w>w^{*}$ or $w<w^{*}$. Asymptotically, the condition for broad resets is thus $w_{\mathrm{fp}}>w^{*}$, where $w_{\mathrm{fp}}$ is the fixed point of $\Phi$. Given the properties of $\Phi$, this means $\Phi\left(w^{*}\right)>w^{*}$. Since the parameter $b$ (spike-triggered adaptation) shifts the curve of $\Phi$ vertically, there is a minimum $b$ above which resets are (at least asymptotically) broad.

When $\Phi$ is continuous (cases $2 \mathrm{~d}$ and $1 \mathrm{~b}$ ), it always has a fixed point (since $\Phi(w)>w+b$ for low $w$ and $\Phi$ converges to a finite limit when $w \rightarrow+\infty$ ), but that fixed point may not be stable. That property depends on all parameter values; in particular, the fixed point is an attraction basin when $b$ or $I$ is large enough (for large $b$, the fixed point is on the plateau of $\Phi$, which implies broad resets). If the fixed point is not stable, then the sequence $\left(w_{n}\right)$ may converge to a periodic orbit or be irregular.

\subsubsection{Bursting}

A bursting response is a sequence of shortly spaced spikes, separated by longer intervals. For the adaptation variable $w$, it corresponds to a periodic orbit, where the period equals the number of spikes per burst. For the adaptation map, $p$-periodic orbits are associated with stable fixed points of $\Phi^{p}$. This situation is illustrated in Fig. 7. Typically, bursting occurs for large reset values $V_{r}$ : the first spike resets the trajectory to a high voltage value, which induces a fast spike, and the adaptation 

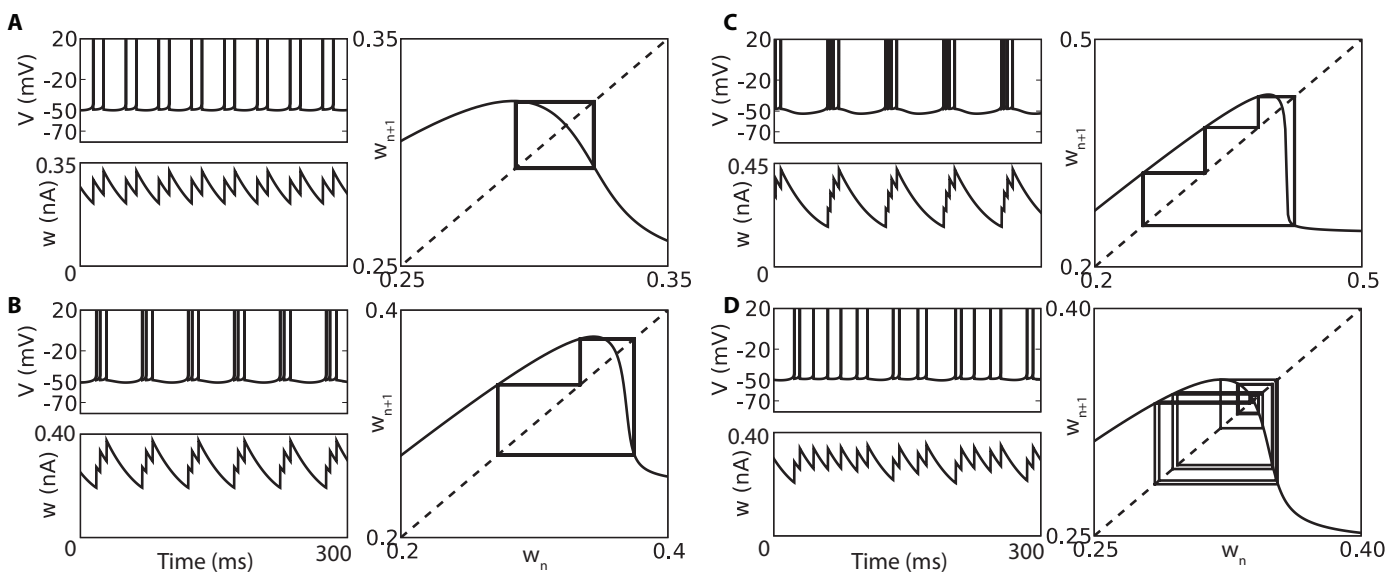

Figure 7: Bursting and chaos. Each panel shows a sample response ( $V$ and $w$ ) from the model, with different values of $V_{r}$ (parameters: $C=281 \mathrm{pF}, g_{L}=30 \mathrm{nS}, E_{L}=-70.6 \mathrm{mV}, V_{T}=-50.4 \mathrm{mV}$, $\left.\Delta_{T}=2 \mathrm{mV}, \tau_{w}=40 \mathrm{~ms}, a=4 \mathrm{nS}, b=0.08 \mathrm{nA}, I=.8 \mathrm{nA}\right)$. A burst with $n$ spikes corresponds to an $n$-periodic orbit under $\Phi$. The last spike of each burst occurs in the decreasing part of $\Phi$, inducing a slower trajectory. A. Bursting with 2 spikes $\left(V_{r}=-48.5 \mathrm{mV}\right)$. B. Bursting with 3 spikes $\left(V_{r}=-47.7\right.$ $\mathrm{mV})$. C. Bursting with 4 spikes $\left(V_{r}=-47.2 \mathrm{mV}\right)$. D. Chaotic spiking $\left(V_{r}=-48 \mathrm{mV}\right)$.

builds up after each spike, until the trajectory is reset above the $V$-nullcline (after the peak of $\Phi$ at $\left.w^{*}\right)$. At that point $d V / d t<0$ and the trajectory must turn in phase space before it spikes, producing a long interspike interval. Thus, the number of spikes per burst increases when $V_{r}$ increases (since $w^{*}$ increases with $V_{r}$ ) and when $b$ decreases. Thus the bifurcation diagram with respect to $V_{r}$ (Fig. 8) shows a period adding structure. Interestingly, when zooming on a transition from $n$ to $n+1$ spikes, a period doubling structure appears, revealing chaotic orbits.

\subsubsection{Chaotic spiking}

The period doubling structure shown in Fig. 8B implies that orbits are chaotic for some parameter values. A sample response of the model for one of those values is shown in Fig. 7D. It results in irregular, unpredictable firing, in response to a constant input current.

\subsection{Phasic spiking}

Phasic spiking or (bursting) can occur in subthreshold regimes $\left(I<I_{\mathrm{rh}}^{I}\right.$ for type I excitability, $I<I_{\mathrm{rh}}^{I I}$ for type II excitability), when there is a stable fixed point and $\mathscr{D} \neq \mathbb{R}$. In that case, the system needs to be destabilized (e.g. a short current pulse, which may be positive or negative, as explained section 2.7). The situation depends on the properties of the attraction basin of the stable fixed point, and can be understood from Fig. 6 . 

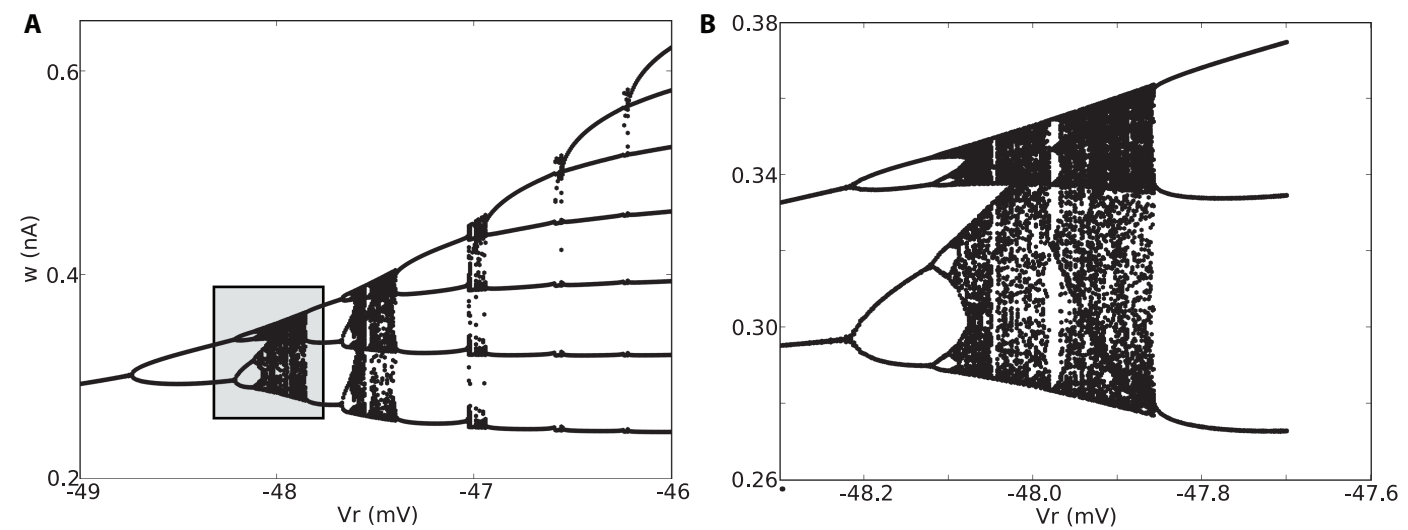

Figure 8: Bifurcation structure with increasing $V_{r}$ (same parameters as in Fig. 7). A. Bifurcation diagram showing a period adding structure (orbits under the adaptation map $\Phi$ with varying values for $V_{r}$ ). Fixed points indicate regular spiking, periodic orbits indicate bursting, dense orbits indicate chaos. B. Zoom on the bifurcation diagram A (as indicated by the shaded box), showing a period doubling structure.

We can distinguish two cases:

1. If $\mathscr{D}=\left(-\infty, w_{\min }\right)\left(\mathrm{C}, \mathrm{D}\right.$ : integrator or mixed regime), then when $V_{-}<V_{r}<V_{+}$there can only be phasic spiking, otherwise tonic spiking is possible. Indeed, if $V_{-}<V_{r}<V_{+}$, then the sequence $\left(w_{n}\right)$ is such that $w_{n+1}>w_{n}+b$, so that it must exit $\mathscr{D}$ in finite time.

2. If $\mathscr{D}=\left(-\infty, w_{\min }\right) \cup\left(w_{\max },+\infty\right)$ (A,B: resonator or mixed regime), then there can only be phasic spiking $\Phi\left(w_{\min }\right)>w_{\max }$, otherwise tonic spiking is possible.

When tonic spiking (or bursting) is possible, then the model is bistable (it can be turned on or off with current pulses).

\section{Discussion}

The adaptive exponential integrate-and-fire model [Brette and Gerstner(2005)] is able to reproduce many electrophysiological features seen in real neurons, with only two variables and four free parameters. Besides, its parameters have a direct physiological interpretation. In the framework of this model, we can define an electrophysiological class as a set of dynamical properties for different values of the input $I$ (for given parameter values). In this paper, we tried to provide a classification of the parameter space as complete as possible, which is summarized for subthreshold dynamics in Fig. 3. The subthreshold dynamics depends only on the ratio of time constants $\left(\tau_{m} / \tau_{w}\right)$ and on the ratio of conductances $\left(a / g_{L}\right)$, but is already non-trivial. The model can have excitability type I or II depending whether it leaves the resting state through a saddle-node or an Andronov-Hopf 
bifurcation. It may act as an oscillator or an integrator depending on the eigenvalues associated to the resting point. It may spike in response to hyperpolarizing currents (rebound), depending on the properties of the attraction basin of the stable fixed point, which is bounded by either a limit cycle or a separatrix.

The spiking dynamics is even more rich, as it also depends on the reset parameters $b$ and $V_{r}$. We related the spike patterns with orbits under a discrete Poincaré map $\Phi$, and found a rich bifurcation structure including even chaos. Regular spiking corresponds to a stable fixed point of $\Phi$, bursting corresponds to periodic orbits under $\Phi$ and irregular spiking corresponds to chaotic orbits under $\Phi$.

Most of the results shown in this paper generalize to two-dimensional spiking models in which the first (membrane) equation is $d V / d t=F(V)+I-w$, where $F$ is a smooth convex function whose derivative is negative at $-\infty$ and infinite at $+\infty$ (in particular, Izhikevich model and the quartic model have these properties). We are currently working on the mathematical proofs of these results in that more general setting and on a more complete picture of the spiking dynamics [Touboul and Brette (2008)]. This work will provide both a dynamical system understanding of the the spiking properties of the model and analytical methods to relate the parameter values with electrophysiological classes. Another interesting line of research is the investigation of the responses of such bidimensional models to time-varying inputs, as was done in [Brette(2004)] for one-dimensional integrate-and-fire models.

\section{Acknowledgements}

We thank Wulfram Gerstner and Richard Naud for discussions. This work was partly supported by funding of the European Union under the grant no. 15879 (FACETS).

\section{References}

[Angelino and Brenner(2007)] Angelino E, Brenner MP (2007) Excitability constraints on voltagegated sodium channels. PLoS Comput Biol 3(9):1751-60

[Badel et al(2008)Badel, Lefort, Brette, Petersen, Gerstner, and Richardson] Badel L, Lefort S, Brette R, Petersen C, Gerstner W, Richardson M (2008) Dynamic IV Curves Are Reliable Predictors of Naturalistic Pyramidal-Neuron Voltage Traces. Journal of Neurophysiology 99(2):656

[Brette and Gerstner(2005)] Brette R, Gerstner W (2005) Adaptive exponential integrate-and-fire model as an effective description of neuronal activity. J Neurophysiol 94:3637-3642

[Brette(2004)] Brette R (2004) Dynamics of one-dimensional spiking neuron models. J. Math. Biol $48(1): 38-56$

[Clopath et al(2007)Clopath, Jolivet, Rauch, Lüscher, and Gerstner] Clopath C, Jolivet R, Rauch A, Lüscher H, Gerstner W (2007) Predicting neuronal activity with simple models of the threshold 
type: Adaptive Exponential Integrate-and-Fire model with two compartments. Neurocomputing 70(10-12):1668-1673

[Fourcaud-Trocme et al(2003)Fourcaud-Trocme, Hansel, van Vreeswijk, and Brunel] FourcaudTrocme N, Hansel D, van Vreeswijk C, Brunel N (2003) How Spike Generation Mechanisms Determine the Neuronal Response to Fluctuating Inputs. Journal of Neuroscience 23(37):11,628

[Gerstner and Kistler(2002)] Gerstner W, Kistler W (2002) Spiking Neuron Models. Cambridge University Press

[Goodman and Brette(2008)] Goodman D, Brette R (2008) Brian: a simulator for spiking neural networks in Python, Frontiers in Neuroinformatics (in preparation)

[Hille(2001)] Hille B (2001) Ion channels of excitable membranes. Sinauer Sunderland, Mass

[Izhikevich(2004)] Izhikevich E (2004) Which model to use for cortical spiking neurons? IEEE Trans Neural Netw 15(5):1063-1070

[Jolivet et al(2008)Jolivet, Kobayashi, Rauch, Naud, Shinomoto, and Gerstner] Jolivet $\quad$ R, Kobayashi R, Rauch A, Naud R, Shinomoto S, Gerstner W (2008) A benchmark test for a quantitative assessment of simple neuron models. J Neurosci Meth 169(2):417-424

[Markram et al(2004)] Markram H, Toledo-Rodriguez M, Wang Y, Gupta A, Silberberg G, Wu C (2004) Interneurons of the neocortical inhibitory system. Nat Rev Neurosci 5(10):793-807

[Lapicque(1907)] Lapicque L (1907) Recherches quantitatives sur l'excitation électrique des nerfs traitée comme une polarisation. J Physiol Pathol Gen 9:620-635

[Naud et al(2008)] Naud R, Macille N, Clopath C, and Gerstner W (2008) Firing Patterns in the Adaptive Exponential Integrate-and-Fire model, Biol. Cyber 2008 (submitted)

[Touboul(2008)] Touboul J (2008) Bifurcation analysis of a general class of nonlinear integrateand-fire neurons. SIAM Appl Math 68:1045-1079

[Touboul and Brette (2008)] Touboul J and Brette R (2008) Spiking dynamics of bidimensional integrate-and-fire neurons (in preparation)

\section{A Oscillations}

In this appendix we calculate the parameter zones where the system oscillates in the rescaled model (3). We then obtain the equations for the original model using the change of variables (5). Damped subthreshold oscillations appear only when the systems has a stable fixed point, i.e. if $\bar{I} \leq(1+$ $\bar{a})(\log (1+\bar{a})-1)$ for $\bar{a}<\frac{1}{\tau_{w}}$ and $\bar{I} \leq\left(1+\frac{1}{\tau_{w}}\right) \log (1+\bar{a})-(1+\bar{a})$ for $\bar{a} \geq \frac{1}{\tau_{w}}$. Furthermore, the 
system will oscillate around the stable equilibrium $v_{-}$if and only if the imaginary part of the eigenvalues of the Jacobian matrix of the system at this point is non-null. This condition can be written at the stable equilibrium $v_{-}$via the discriminant $\delta$ defined by:

$$
\delta\left(v_{-}\right)=\left(e^{v_{-}}-1+\frac{1}{\overline{\tau_{w}}}\right)^{2}-4 \frac{\bar{a}}{\overline{\tau_{w}}} .
$$

The system will oscillate around the stable fixed point $v_{-}$if and only if $\delta<0$. To invert this inequality, we compute the zones where we have

$$
\left(x-1+\frac{1}{\overline{\tau_{w}}}\right)^{2}-4 \frac{\bar{a}}{\overline{\tau_{w}}}<0
$$

and check that a solution $v_{-}$exists. There exists a $v_{-}$such that $e^{v_{-}}=x$ if and only if $0<x \leq(1+\bar{a})$, since $v_{-}<\log (1+\bar{a})$.

The solution of (14) is $x \in\left\{x_{-}, x_{+}\right\}$where

$$
x_{ \pm}=\frac{\overline{\tau_{w}}-1 \pm 2 \sqrt{\bar{a} \overline{\tau_{w}}}}{\overline{\tau_{w}}}
$$

First of all we are interested in the apparition of oscillations in the type I case. We know that when the input current $I$ is close to the rheobase current $I_{\mathrm{rh}}^{I}$ given by (7), the system returns monotonously to the resting potential. The system begins to oscillate when there exist solutions to the equation $e^{v_{-}}=x_{+}$. It is straightforward to check that $x_{+}$is always lower than $(1+\bar{a})$, since this condition is equivalent to the condition $\left(\sqrt{\overline{\tau_{w}} \bar{a}}+1\right)^{2} \geq 0$, which is always true. The condition $x_{+}>0$ is satisfied on the parameter zone

$$
\left\{\left(\overline{\tau_{w}}, \bar{a}\right) ; \overline{\tau_{w}}>1 \text { or } \overline{\tau_{w}}<1 \text { and } \bar{a}>\frac{1}{4 \overline{\tau_{w}}}\left(1-\overline{\tau_{w}}\right)^{2}\right\}
$$

In this zone, oscillations occur when the current $\bar{I}$ is below $\overline{I_{+}}$, where:

$$
\overline{I_{+}}=(1+\bar{a}) \log \left(\frac{\overline{\tau_{w}}-1+2 \sqrt{\bar{a} \overline{\tau_{w}}}}{\overline{\tau_{w}}}\right)-\frac{\overline{\tau_{w}}-1+2 \sqrt{\bar{a} \overline{\tau_{w}}}}{\overline{\tau_{w}}}
$$

Hence it appears in the type I excitable case. After the Bogdanov-Takens point, the equilibrium associated with $x_{+}$is unstable, hence does not give rise to damped subthreshold oscillations.

It is easy to show that $1 /\left(4 \overline{\tau_{w}}\right)\left(1-\overline{\tau_{w}}\right)^{2}<1 / \overline{\tau_{w}}$. When $\bar{a}=1 / \overline{\tau_{w}}$, we have $\overline{I_{+}}=(1+\bar{a})(\log (1+$ $\bar{a})-1)$, which is the current at the Bogdanov-Takens bifurcation point. This result was predictible since around the saddle node bifurcation the system does not oscillate around the fixed point and around the Andronov-Hopf bifurcation the system does oscillate, and these two curves meet at the Bogdanov-Takens point. Furthermore, after the Bogdanov-Takens point, the equilibrium associated with $x_{+}$is no more stable, hence damped subthreshold oscillations associated with this separatrix only appear in the type I excitable case.

The oscillations possibly disappear when a solution to $e^{v_{-}}=x_{-}$exists. These solutions exist when $\overline{\tau_{w}}>1$ and $\bar{a} \leq \frac{\overline{\tau_{w}}}{4}\left(1-\frac{1}{\overline{\tau_{w}}}\right)^{2}$. Thus, oscillations disappear when $\bar{I}<\overline{I_{-}}$, where: 


$$
\overline{I_{-}}=(1+\bar{a}) \log \left(\frac{\overline{\tau_{w}}-1-2 \sqrt{\bar{a} \overline{\tau_{w}}}}{\overline{\tau_{w}}}\right)-\frac{\overline{\tau_{w}}-1-2 \sqrt{\bar{a} \overline{\tau_{w}}}}{\overline{\tau_{w}}}
$$

With the original parameters, the expression of $I_{ \pm}$reads:

$$
\begin{aligned}
I_{ \pm}=\left(g_{L}+a\right) \Delta_{T} \log \left(\frac{g_{L} \tau_{w}-C \pm 2 \sqrt{a C \tau_{w}}}{g_{L} \tau_{w}}\right) & \\
& -\Delta_{T} \frac{g_{L} \tau_{w}-C \pm 2 \sqrt{a C \tau_{w}}}{\tau_{w}}-\left(g_{L}+a\right)\left(E_{L}-V_{T}\right)
\end{aligned}
$$

Hence, grouping the cases as a function of $\overline{\tau_{w}}$ and $\bar{a}$, we have:

- For $\overline{\tau_{w}}<1$ :

- The system always returns monotonously to equilibrium when $\bar{a}<\frac{1}{4 \overline{\tau_{w}}}\left(1-\overline{\tau_{w}}\right)^{2}$ or when $\bar{a}>\frac{1}{4 \overline{\tau_{w}}}\left(1-\overline{\tau_{w}}\right)^{2}$ and $\bar{I}>\overline{I_{+}}$.

- The system oscillates around equilibrium $\bar{a}>\frac{1}{4 \overline{\tau_{w}}}\left(1-\overline{\tau_{w}}\right)^{2}$ and $\bar{I}<\overline{I_{+}}$.

- For $\overline{\tau_{w}}>1$ :

- the system oscillates for any $\bar{I} \in\left(\overline{I_{-}}, \overline{I_{+}}\right)$when $\bar{a}<\frac{1}{4 \overline{\tau_{w}}}\left(1-\overline{\tau_{w}}\right)^{2}$ and for any $\bar{I}<\overline{I_{+}}$if $\bar{a}>\frac{1}{4 \bar{\tau}_{w}}\left(1-\overline{\tau_{w}}\right)^{2}$.

- otherwise it returns monotonously to its resting state.

When the system oscillates, the oscillation (angular) frequency is given by $\bar{\omega}=-\delta$, which, in the low-voltage approximation (far from $V_{T}$ ), reads:

$$
\bar{\omega} \approx 4 \frac{\bar{a}}{\overline{\tau_{w}}}-\left(1-\frac{1}{\overline{\tau_{w}}}\right)^{2}
$$

When the system oscillates, the time constant of the decay is the inverse of the opposite of the real part of the eigenvalues, which is $-1 / 2\left(e^{v_{-}}-1-\frac{1}{\tau_{w}}\right)$. With the original parameters, the time constant is thus:

$$
\left(\frac{1}{2}\left(\frac{1}{\tau_{m}}+\frac{1}{\tau_{w}}-\frac{1}{\tau_{m}} e^{\frac{V-V_{T}}{\Delta_{T}}}\right)\right)^{-1}
$$

and in the low-voltage approximation it is simply

$$
2 \frac{\tau_{m} \tau_{w}}{\tau_{m}+\tau_{w}}
$$

$\mathrm{RR} \mathrm{n}^{\circ} 6563$ 


\section{B Overshoot}

As discussed in section 2.5.2, the response of the neuron to a current step can present an overshoot when the coefficient of the slower exponential term is negative. In this section we show that in the low-voltage approximation $\left(V \ll V_{T}\right)$, there is an overshoot if and only if $\tau_{m}<\tau_{w}$ and there is no oscillation, thus, in the mixed mode regime (Fig. 3).

Indeed, in the low voltage approximation, the dynamics is linear and is governed by the operator:

$$
L=\left(\begin{array}{cc}
-1 & -1 \\
\frac{\bar{a}}{\overline{\tau_{w}}} & -\frac{1}{\overline{\tau_{w}}}
\end{array}\right)
$$

which can be diagonalized. The overshoot appears only when the eigenvalues are real. In this case, the voltage response to a short pulse (dirac) is a sum of two exponential functions $v(t)=$ $\alpha e^{-t / \tau_{1}}+\beta \exp ^{-t / \tau_{2}}$ (we set the resting potential to 0 ) where $\frac{-1}{\tau_{1}}$ and $\frac{-1}{\tau_{2}}$ are the two real eigenvalues of $L$. The coefficient of the slower exponential term is

$$
\frac{\varepsilon}{2 \delta}\left(\sqrt{\delta}\left(1-\overline{\tau_{w}}\right)+\delta\right)
$$

with $\delta=\left(1-\overline{\tau_{w}}\right)^{2}-4 \bar{a} \overline{\tau_{w}}$. We now write the negativity condition of this coefficient:

$$
\sqrt{\delta}\left(1-\bar{\tau}_{w}\right)+\delta<0 \Leftrightarrow 1-\bar{\tau}_{w}<-\sqrt{\delta}
$$

A necessary condition for this inequality to be satisfied is $\overline{\tau_{w}}>1$. In this case, the condition reads:

$$
\left(1-\overline{\tau_{w}}\right)^{2}>\delta=\left(1-\overline{\tau_{w}}\right)^{2}-4 \bar{a} \overline{\tau_{w}}
$$

which is always true since $\bar{a} \bar{\tau}_{w}>0$. Hence the overshoot appears in the low voltage approximation (far from threshold) when $\overline{\tau_{w}}>1$, i.e., when $\tau_{m}<\tau_{w}$.

\section{Separatrix}

\section{C.1 Position of the stable manifold}

Some information about the stable manifold of the saddle fixed point can be obtained from the nullclines (when these intersect). The nullclines cut the plane in 5 connected zones, which we call North, South, West, East and Center, as shown in Fig. 1. The stable manifold consists in two trajectories which converge to the saddle fixed point. Near the saddle point, these two trajectories must lie in the North and South zone, or in the Center and East zones.

First we remark that all the trajectories starting from the East zone must spike. Indeed, in that region, $V$ increases and $w$ decreases, until it crosses the w-nullcline horizontally and enters the South zone. From that point, $V$ keeps on increasing and $w$ increases, which implies that the trajectory can only remain in the South zone or enter the East zone. However, the direction of the vector fields along the border does not allow crossing from South to East. Therefore, the trajectory will remain 
in the South zone and will spike. It follows that no part of the stable manifold can be in the East zone. Therefore it has to be locally in the North and South zones. By following the manifold from the saddle point to the North, we can see that $V$ and $w$ increase and, since the manifold cannot enter the East zone, it remains in the North zone and goes to infinity. In practice, it is in fact very close (but slightly to the left) of the V-nullcline, as shown in Fig. 4.

By following the manifold from the saddle point to the South, we can see that it has the same orientation as in the North zone, as long as it remains in the South zone. It may however cross the w-nullcline (Fig. 4D), and possibly the V-nullcline again (Fig. 4B).

\section{C.2 Asymptotic behavior of the solutions}

To understand whether the stable manifold can cross the w-nullcline and possibly the V-nullcline, we study the asymptotic behavior of the solutions when $t \rightarrow-\infty$. Here again we consider the rescaled model (3). The idea is the following: if the manifold goes to $-\infty$ (for $V$ ), then the exponential term vanishes and its approximated dynamics can be solved analytically. Thus, in the following we shall assume that the manifold does not cross the V-nullcline. In that case, the voltage $V(t)$ of the manifold, seen as a solution of the system, goes to $-\infty$ as $t \rightarrow-\infty$, and we will look for possible contradictions.

Asymptotically, the differential equations satisfied by a given solution $(v, w)$ of the rescaled model can be approximated by:

$$
\begin{cases}\dot{v} & =-v-w+I \\ \overline{\tau_{w}} \dot{w} & =\bar{a} v-w\end{cases}
$$

When $t \rightarrow-\infty$, the solutions of the linear system either spiral around the fixed point (complex eigenvalues) or align asymptotically to the direction of eigenvector associated to the smallest negative eigenvalue of the matrix $L$ governing the dynamics of the linear system (16):

$$
L=\left(\begin{array}{cc}
-1 & -1 \\
\bar{a} & -\frac{1}{\tau_{w}}
\end{array}\right)
$$

If the eigenvalues of this matrix are complex, i.e., when $\bar{a}>\frac{\left(\bar{\tau}_{w}-1\right)^{2}}{4 \bar{\tau}_{w}}$, then the solutions spiral around the fixed point. Therefore the trajectories cross the V-nullcline, which contradicts our initial hypothesis. Thus when $\bar{a}>\frac{\left(\bar{\tau}_{w}-1\right)^{2}}{4 \bar{\tau}_{w}}$ (resonator regime), the stable manifold crosses both nullclines.

If the eigenvalues are real, the trajectories of the linear system align asymptotically to the direction of the lower eigenvalue

$$
\lambda_{-}=-\frac{1}{2 \bar{\tau}_{w}}\left(\overline{\tau_{w}}+1+\sqrt{\left.\left(\overline{\tau_{w}}-1\right)^{2}-4 \overline{\tau_{w}} \bar{a}\right)}\right.
$$

This eigenvalue is always strictly negative hence solutions will diverge when $t \rightarrow-\infty$. The eigenvector associated with this eigenvalue is: 


$$
\left(\begin{array}{c}
\frac{2 \overline{\tau_{w}}}{1-\overline{\tau_{w}}+\sqrt{\left(\overline{\tau_{w}}-1\right)^{2}-4 \bar{a} \overline{\tau_{w}}}} \\
1
\end{array}\right)
$$

The slope of that eigenvector is always below -1 , so that (linearized) trajectories do not cross the $\mathrm{V}$-nullcline. However they can cross the w-nullcline when the slope of the eigenvector is smaller than $\bar{a}$, i.e.:

$$
\frac{1-\overline{\tau_{w}}+\sqrt{\left(\overline{\tau_{w}}-1\right)^{2}-4 \bar{a} \bar{\tau}_{w}}}{2 \bar{\tau}_{w}}<\bar{a}
$$

and this condition is satisfied when $\bar{a}>\frac{1}{2}\left(\frac{1}{\tau_{w}}-1\right)$. Assuming $\bar{a}>0$, the inequality is always true if $\overline{\tau_{w}}>1$; when when $\overline{\tau_{w}}<1$, the inequality is never true given that the eigenvalues are real $(\bar{a}<$ $\left.\frac{\left(\overline{\tau_{w}}-1\right)^{2}}{4 \overline{\tau_{w}}}\right)$.

In summary, the stable manifold crosses both nullclines when $\bar{a}>\frac{\left(\overline{\tau_{w}}-1\right)^{2}}{4 \overline{\tau_{w}}}$ (resonator regime), and it crosses at least the w-nullcline when $\overline{\tau_{w}}>1$. 


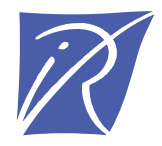

\section{Unité de recherche INRIA Sophia Antipolis 2004, route des Lucioles - BP 93 - 06902 Sophia Antipolis Cedex (France)}

Unité de recherche INRIA Futurs : Parc Club Orsay Université - ZAC des Vignes 4, rue Jacques Monod - 91893 ORSAY Cedex (France) Unité de recherche INRIA Lorraine : LORIA, Technopôle de Nancy-Brabois - Campus scientifique 615, rue du Jardin Botanique - BP 101 - 54602 Villers-lès-Nancy Cedex (France)

Unité de recherche INRIA Rennes : IRISA, Campus universitaire de Beaulieu - 35042 Rennes Cedex (France)

Unité de recherche INRIA Rhône-Alpes : 655, avenue de l'Europe - 38334 Montbonnot Saint-Ismier (France)

Unité de recherche INRIA Rocquencourt : Domaine de Voluceau - Rocquencourt - BP 105 - 78153 Le Chesnay Cedex (France)

Éditeur

INRIA - Domaine de Voluceau - Rocquencourt, BP 105 - 78153 Le Chesnay Cedex (France)

http://www.inria.fr

ISSN 0249-6399 